\title{
A New Triangular Matching Approach for Latent Palmprint Identification
}

\author{
José Hernández-Palancar, Alfredo Muñoz-Briseño, and Andrés Gago-Alonso \\ Advanced Technologies Application Center (CENATAV), Cuba \\ $\{$ jpalancar, amunoz, agago\}@cenatav.co.cu
}

\begin{abstract}
Palmprint identification is still considered as a challenging research line in Biometrics. Nowadays, the performance of this techniques highly depends on the quality of the involved palmprints, specially if the identification is performed in latent palmprints. In this paper, we propose a new feature model for representing palmprints and dealing with the problems of missing and spurious minutiae. Moreover, we propose a novel verification algorithm based in this feature model, which uses a strategy for finding adaptable local matches between substructures obtained from images. In experimentation, we show that our proposal achieves highest scores in latent palmprint matching, improving some of the best results reported in the literature.
\end{abstract}

\section{Introduction}

In the last years, the interest in recognition of persons by their palmprints has grown. There are some scientific studies that ensure the uniqueness of the palmprint of a person and the much fancied stability over time or age [1]. In this sense such technique is much better than others, specially in forensic cases where other biometric information is not available.

Palmprints are marks produced by the contact of the palm of the hand with a surface. These marks reflect the different patterns formed by the ridges that are visible in the epidermis. Most of the verification approaches use minutiae as basis for representing palmprints and checking mutual matches. However, the features extraction is still a challenging problem since the possibility of finding false minutiae always exists [6].

There are not many published articles about the topic, specially in the latent case since palmprint identification is considered as a challenging problem. Until today, few works on latent-to-full palmprint matching have been done. One of the first relevant proposed methods was based on a feature called MinutiaCode [6]. However, this proposal is time consuming and not robust to distortions. Another recent works use radial triangulations in order to extract features [9,10]. Even when the use of radial triangulations increase the accuracy, the features extracted from them are still affected by stretching in the skin. Finally, Jain et al. 7] proposed a method based on minutiae clustering and minutiae match propagation.

J. Ruiz-Shulcloper and G. Sanniti di Baja (Eds.): CIARP 2013, Part II, LNCS 8259, pp. 294-301 2013.

(C) Springer-Verlag Berlin Heidelberg 2013 
One of the most relevant contribution of this paper is to present the results of our palmprint matching algorithm dealing with low quality or distorted palmprint images. It is important to note that unlike other approaches 67910, our proposal do not uses any enhancement method in the minutiae extraction process. Our novel matching algorithm uses a representation proposed in literature [5], called expanded triangle set, which is based on minutia triplets obtained from Delaunay triangulation and other redundant ones for reducing the negative effect of structural distortions. Expanded triangle set was previously used for fingerprint indexing and retrieving tasks [5], whereas it is currently used for palmprint matching, in our research. In our matching step, we propose a new strategy to find local matches between substructures formed in the palmprints.

This work is organized as follows. In Section 2 some concepts and definitions necessary to understand our proposal, are described. The Section 3 is dedicated to define our palmprint representation and to describe the process of features extraction. In Section 4, is defined a matching algorithm that uses the features extracted. In Section 5] some experimental results that validate the accuracy of our proposal, are shown. Finally, Section 6 contains the conclusions.

\section{Background}

In this section, we present some basic concepts and a general scheme of palmprint matching algorithms. Thus, we declare the necessary background for understanding our proposal and the rest of the paper. Finally, we describe the Delaunay triangulation and its properties, considering that this kind triangulation is used in many contexts for representing ridges patterns, including our approach.

\subsection{The Expanded Triangle Set}

In general, a triangulation of a set of points, $P=\left\{p_{1}, p_{2}, \ldots, p_{N}\right\}$, in the plane is the set of triangles that conforms a maximal planar subdivision whose vertex set is $P$. A maximal planar subdivision is a subdivision $S$ such that no edge connecting two vertices can be added to $S$ without destroying its planarity [2]. Especially, a triangulation of $P$ is a Delaunay triangulation if and only if every triangle $\triangle P_{i} P_{j} P_{k}$ that belongs to $T$ satisfies that its circumcircle contains no other point of $P$ [2]. The Delaunay graph of a Delaunay triangulation $T$ is defined as a tuple $G=\langle P, E\rangle$ where $P$ is the set of planar points that originated $T$, and $E$ is the set of edges that conforms the triangles of $T$; each edge has a single occurrence in $E$.

Delaunay triangulations have some theoretical properties, which are very useful for palmprint matching. However, it must be highly affected, when the extraction method fails to find a minutia [5]. For example, in Fig. 1(a), we can see a Delaunay triangulation of a set of points. In Fig. 1(b), we can appreciate major structural changes in the same triangulation when removing the vertex $p$. In literature there is a proposal that introduces an interesting criterion for selecting minutia triplets called expanded triangle set [5], which is defined as follows. 
Definition 1 (Triangular hull). Let $p_{i}$ be a point of $P$. The set $N_{i}=$ $\left\{p_{j} \mid\left\{p_{i}, p_{j}\right\} \in E\right\}$ denoted the point set formed by all the adjacent vertices of $p_{i}$ in the Delaunay graph $G$. The triangular hull of $p_{i}$ is defined as the Delaunay triangulation of the planar point set $N_{i}$, and it is denoted by $H_{i}$.

Definition 2 (Expanded triangle set). The expanded triangle set of $P$ is defined as $R=T \cup H_{1} \cup H_{2} \cup \ldots \cup H_{N}$.

The set $R$ includes the triangles in the Delaunay triangulation of $P$ and any triangle in the triangular hulls of the points in $P$. Despite the fact that $|R|$ is greater than $|T|$, the number of triangles of $|R|$ is still linear with respect to $N$ [5. This is very desirable if we consider that the sets $R$ will be used as a representation for palmprints in verification tasks.



(a)
Delaunay triangulation, $T$



(b)

Delaunay triangulation without $p_{i}, H_{i}$

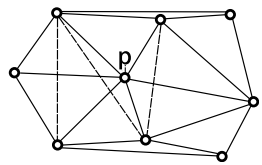

(c) Expanded triangle set, $R=T \cup H_{i}$

Fig. 1. Triangle set examples

The advantage of the set $R$ is that it contains all of the Delaunay triangles that are formed when each minutia is eliminated individually. In this way, we ensure that even when the extraction method fails to find a minutia, some of the matchings will be found. For example, Fig. 1(c) shows the expanded set of the points including $p_{i}$. As we can see, Fig. 1(c) has corresponding triangles with both, Fig. 1(a) and Fig. 1(b) due to the use of the expanded triangle set. In this paper, the expanded triangle set of minutiae is used for representing palmprints in verification tasks.

\subsection{Palmprint Matching}

In general, we can say that a palmprint matching algorithm compares two palmprints and returns either a degree of similarity or a binary decision. Until today, matching palmprints is still a topic of interest due to the noise and distortions in palm images that can be produced by scars, creases and cuts.

In our case, the palmprints are described as vectors of minutiae were each one can have some attributes. The most commonly used attributes are the coordinates, direction and type of minutiae.

More formally, let $T_{1}=\left\{m_{1}, m_{2}, \ldots, m_{n}\right\}$ and $T_{2}=\left\{m_{1}, m_{2}, \ldots, m_{m}\right\}$ be minutia vectors that describe two palmprints, where $m_{i}=\left(x_{i}, y_{i}\right)$. In order to 
obtain a similarity score between $T_{1}$ and $T_{2}$, the matching algorithms try to establish similarities between their minutiae. A later step of consolidation consists on computing a global score based on the matches found among minutiae.

\section{$3 \quad$ Feature Extraction Step}

In this section, we propose a new feature model for representing palmprints, using the expanded triangle set obtained from minutiae, see section 2.1. We are considering that the minutiae extraction process for full palmprints is carried out by any algorithm reported in the state-of-the-art and marked manually for latent palmprints.

Let $P=\left\{p_{1}, p_{2}, \ldots, p_{N}\right\}$ be the set containing all the planar points representing the minutiae in a palmprint $F$. Let $R$ be the expanded triangle set of $P$, and let $t \in R$ be a triangle, which represents a minutia triplet. Let $m_{1}=\left(x_{1}, y_{1}\right)$, $m_{2}=\left(x_{2}, y_{2}\right)$, and $m_{3}=\left(x_{3}, y_{3}\right)$ be the three points of $t$, with their corresponding planar coordinates, which are sorted in ascending order regarding the length of the opposite side.

The feature vector associated to $t$ in the palmprint $F$ is denoted by $f(t)$, and it is defined as follows

$$
f(t)=\left(s_{t}, \beta_{1}, \beta_{2}, \beta_{3}, r_{1}, r_{2}, r_{3}, d_{1}, d_{2}, d_{3}\right),
$$

where $s_{t}$ is the triangle sign, $\beta_{i}$ are the relative directions of $m_{i}$ with respect to his opposite side in $t, r_{i}$ are the ridge counter between minutiae and $d_{i}$ represent the length of the sides of the triangle. These components are formally defined as follows. The twice signed area of $t$ is calculated using the following mathematical expression

$$
A_{t}=x_{1}\left(y_{2}-y_{3}\right)+x_{2}\left(y_{3}-y_{1}\right)+x_{3}\left(y_{1}-y_{2}\right) .
$$

Using $A_{t}$, we define the triangle sign of $t$ as $s_{t}=0$ if $A_{t}<0$; otherwise $s_{t}=1$. This feature is invariant to rotation and is included in order to discard possible correspondences between similar palmprints of different hands (left and right).

We define $d_{i}$ as the Euclidean distance between the corresponding minutiae $m_{j}$ and $m_{k}$. Finally, the ridge counter $r_{i}$ is defined as the number of ridges crossed by the segment joining the pair of minutiae. We verify the statistical behavior presented in [5]; therefore, we also remove from $R$ those triangles with at least one value outside the interval, $0 \leq r_{i}<16$.

The feature vectors presented in this section can be represented as a function $f: R \rightarrow \Phi$ called feature function, where the set $\Phi=K_{1} \times K_{4}^{3} \times K_{4}^{3} \times \mathbb{R}^{3}$, assuming $K_{n}=\left\{0,1, \ldots, 2^{n}-1\right\}$ represents the feature space. Thus, we are able to define the formal representation of a palmprint $F$, which is used in this paper.

Definition 3 (The feature model). Let $F$ be a palmprint. The model of $F$ is defined as a triplet $M=\langle P, R, f\rangle$, where $P$ is the planar point set representing the minutiae of $F, R$ is the expanded triangle set of $P$, and $f$ is the function $f: R \rightarrow \Phi$, see (1). 
This feature model is used during the matching step for representing the involved palmprints. The described features, combined with the mechanism defined in section 4 to reduce the negative effects of noise, show a good performance when they are used in identification tasks, see section 5 .

\section{Matching Step}

In this step, we obtain a similarity value between two models $M_{p}=\left\langle P_{p}, R_{p}, f_{p}\right\rangle$ and $M_{q}=\left\langle P_{q}, R_{q}, f_{q}\right\rangle$. In order to do this, we present the following.

Let

$$
f\left(t_{l}\right)=\left(s_{t l}, \beta_{1 l}, \beta_{2 l}, \beta_{3 l}, r_{1 l}, r_{2 l}, r_{3 l}, d_{1 l}, d_{2 l}, d_{3 l}\right)
$$

with $l \in\{q, p\}$, be the two feature vectors of two triangles $t_{p} \in R_{p}$ and $t_{q} \in R_{q}$, we say that $t_{p}$ and $t_{q}$ are corresponding if the following geometric constraints are fulfilled:

$$
\begin{aligned}
s_{t p} & =s_{t q}, \\
\left|\beta_{i p}-\beta_{i q}\right| & \leq \delta_{\beta}, \\
\left|r_{i p}-r_{i q}\right| & \leq \delta_{r}, \\
\left|d_{i p}-d_{i q}\right| & \leq \delta_{d},
\end{aligned}
$$

for all $i \in\{1,2,3\}$, where $\delta_{\beta}, \delta_{r}$, and $\delta_{d}$ are predefined thresholds empirically set to 3 .

Let $t_{p}\left(m_{1 p}, m_{2 p}, m_{3 p}\right)$ and $t_{q}\left(m_{1 q}, m_{2 q}, m_{3 q}\right)$ be two corresponding triangles. We define their correlation tuples as $c t_{i}=\left(\alpha_{i}, \overline{m_{i p} m_{j p}}, \overline{m_{i q} m_{j q}}\right)$ with $j=1$ if $i=3$ and $j=i+1$ otherwise; were $\alpha_{i}$ represents the normalized difference between the $\mathrm{i}$-th interior angles of $t_{p}$ and $t_{q}$, and $\overline{m_{i p} m_{j p}}, \overline{m_{i q} m_{j q}}$ are segments of the triangles. Interior angle is defined as the angle inside two adjacent sides of a triangle.

The process followed to obtain the value of $\alpha_{i}$, is very similar to that presented by Chikkerur et al. [3], to obtain the similarity between an edge that connects two minutiae of an impression and one edge joining two minutiae of other fingerprint.

Let $R_{p}$ and $R_{q}$ be two triangles sets, we define the set $T\left(R_{p}, R_{q}\right)=$ $\left\{c t_{1}, c t_{2}, \ldots, c t_{n}\right\}$ as the union of all the correlation tuples of every corresponding triangle between $R_{p}$ and $R_{q}$.

In our matching step, we use a reduced set $\operatorname{Tr}\left(R_{p}, R_{q}\right)=\left\{c t_{1}, c t_{2}, \ldots, c t_{r}\right\}$ that contains only the correlation tuples whose value of $\alpha_{i}$ are equal to the statistic mode in $T\left(R_{p}, R_{q}\right)=\left\{c t_{1}, c t_{2}, \ldots, c t_{n}\right\}$, for the values of $\alpha_{i}$ of every $c t_{i}$. The main goal of this process is finding the most probable value of relative rotation between the matched models and using only the correlation tuples that are consequent with this.

With the reduced set $\operatorname{Tr}\left(R_{p}, R_{q}\right)$ we construct a similarity graph $G_{s}=$ $\langle V, E, L, s, l\rangle$ where $s: E \rightarrow \mathbb{R}$ is a similarity function that assign a value to every edge, $l: P_{i} \times P_{j} \rightarrow L$ is a labeling function given two vertices and $L$ is a set of vertex labels. $s$ is a similarity function that represents in fuzzy terms the grade of closeness between the two segments $\overline{m_{i p} m_{j p}}$ and $\overline{m_{i q} m_{j q}}$ that originated a edge in $G_{s}$. Similar functions had been used in other fingerprint recognition approaches [34. 
In algorithm 1, the generation of $G_{s}$ is described. For each $c t_{i}=$ $\left(\alpha_{i}, \overline{m_{i p} m_{j p}}, \overline{m_{i q} m_{j q}}\right) \in \operatorname{Tr}$ two vertices that represent the mutual match between $m_{i p} \Longleftrightarrow m_{i q}$ and $m_{j p} \Longleftrightarrow m_{j q}$, are generated. If these vertices are not in $V$, they are added. Also, a new edge that represents the mutual match between segments $\overline{m_{i p} m_{j p}}$ and $\overline{m_{i q} m_{j q}}$ is added to $E$. In this way, we have a graph that represents matches between points of the models $M_{p}$ and $M_{q}$, weighted with a similarity function. The graph $G_{s}$ may be not connected.

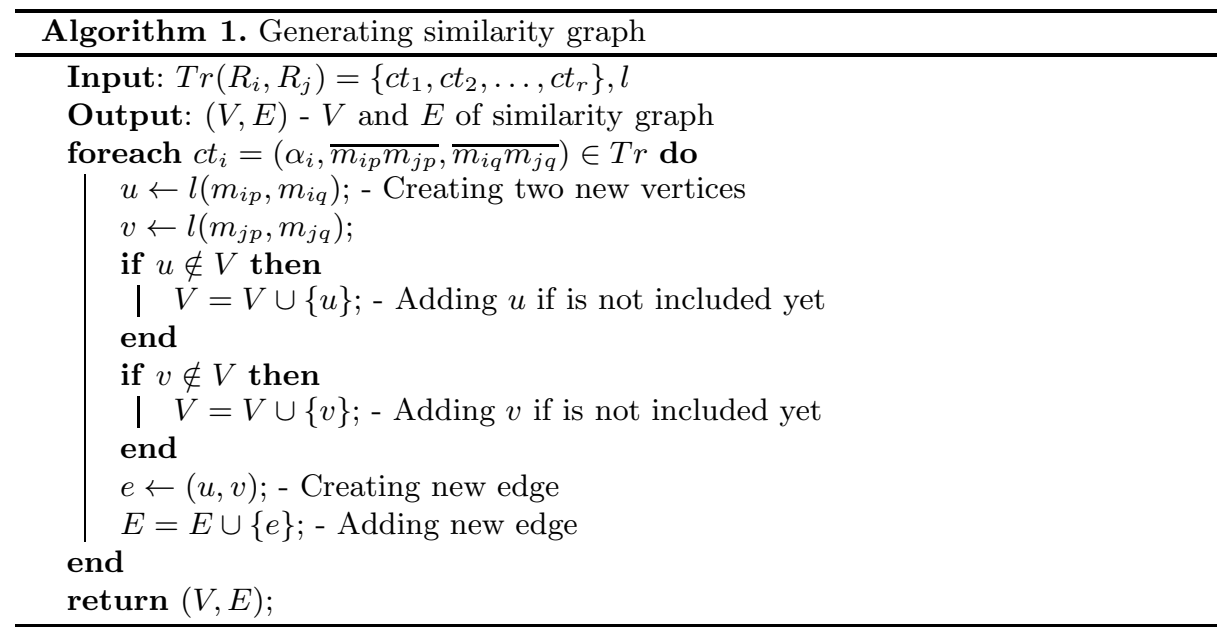

In order to find the spanning tree of every connected components of $G_{s}$ with the higher value of similarity in their edges, we applied the Kruskal algorithm to $G_{s}$. This is a well known method to find a minimum (or maximum in our case) spanning forest of disconnected graphs. Unlike the proposal presented by Zhu et al. 11] based on the Prim algorithm, our solution is superior and it has not been reported in previous works.

Let $\left\{F_{1}, F_{2}, \ldots, F_{n}\right\}$ be the set of spanning trees returned by the Kruskal algorithm, sorted in descending order by the amount of edges. We implement a strategy to merge $F_{1}$ and $F_{2}$ by trying to add a virtual edge $e_{v}$ between then. This virtual edge must complain with some geometric restrictions. If this process is successful then $F_{1}=F_{1} \cup F_{2} \cup\left\{e_{v}\right\}, F_{2}$ is eliminated and $F_{i-1} \leftarrow F_{i}, \forall i, 3<$ $i<n, n \leftarrow n-1$. This process is repeated while $F_{1}$ and $F_{2}$ can be merged.

Finally, the similarity value between between models $M_{p}=\left\langle P_{p}, R_{p}, f_{p}\right\rangle$ and $M_{q}=\left\langle P_{q}, R_{q}, f_{q}\right\rangle$ is given by the following expression:

$$
\operatorname{similarity}\left(M_{p}, M_{q}\right)=\frac{\operatorname{sim} \times|V|}{\min \left(\left|P_{p}\right|,\left|P_{q}\right|\right)}
$$

where $|V|$ is the number of vertices in the similarity graph $G_{s},\left|P_{p}\right|$ and $\left|P_{q}\right|$ are the cardinalities of $P_{p}$ and $P_{q}$ respectively, and sim is the sum of the weights of every edge of $F_{1}$. 


\section{$5 \quad$ Experimental Results}

In our experiments, a dataset in which some other approaches were tested 6910 , was used. This dataset is conformed by 22 latent palmprints from real forensic cases and 8680 full palmprints from criminal investigation field, captured by Beijing Institute of Criminal Technology in China. All the impressions have a resolution of $500 \mathrm{ppi}$. In the case of latent palmprints, the minutiae were manually extracted by forensic chinese experts. On the other hand, the minutiae of the 8680 full palmprints were extracted automatically using the VeriFinger $4.2[8$, and we did not used any palmprint enhancement process.

Table 1. Comparison results of identification rate

\begin{tabular}{|c|c|c|c|}
\hline \multirow{2}{*}{ Algorithm } & \multicolumn{3}{|c|}{ Identification Rate } \\
\cline { 2 - 4 } & Rank-1 & Rank-10 & Rank-20 \\
\hline Jain and Feng [6] & $67 \%$ & $73 \%$ & $80 \%$ \\
\hline Wang et al. [9] & $63 \%$ & $68 \%$ & $72 \%$ \\
\hline Wang et al. [10 & $69 \%$ & $78 \%$ & $\mathbf{8 2} \%$ \\
\hline Our proposal & $\mathbf{7 7 \%} \%$ & $\mathbf{8 2} \%$ & $\mathbf{8 2} \%$ \\
\hline
\end{tabular}

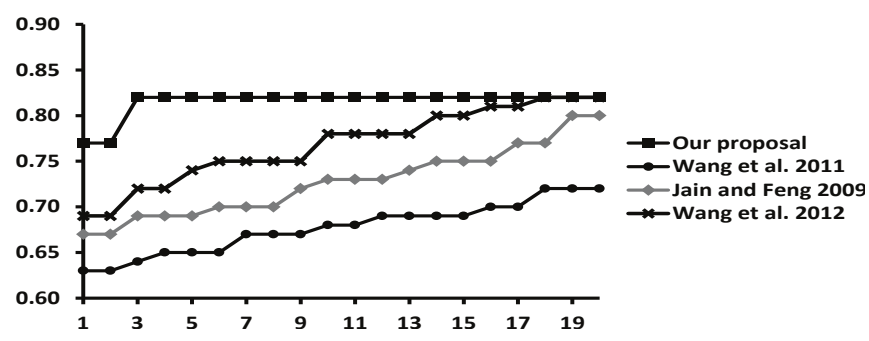

Fig. 2. Comparison of methods using CMC curves

Using the described dataset we compared our latent-to-full palmprint verification algorithm with three other proposals found in the state-of-the-art.

Comparison results of identification rate are shown in Table 1 . For each identification rate, the higher reached value is highlighted in bold. As we can see, in almost every case our method outperforms the other proposals. In Figure 2 , CMC curves of our algorithm and other state-of-the-art methods are shown. In this graphic, the higher accuracy of our proposal is evidenced,especially for rank-1, where we have eight percentage points over the best second algorithm. This same algorithm achieves the $82.2 \%$ of identification rate at rank-18, while our algorithm obtains the same value at rank-3.

\section{Conclusions}

Many of the reported palmprint matching algorithms are highly affected when they are used to compare low-quality and distorted images or latent palmprints 
captured at uncontrolled context. The feature model and the novel matching algorithm, proposed in this paper, can be considered as a promising approach for palmprint identification in such context, dealing with the problems of missing and spurious minutiae, and other noises. Experimental results show that our proposal achieves high accuracy in latent palmprint matching tasks, outperforming other state-of-the-art proposals. We did not use any preprocessing or enhancement method to reduce the number of false minutiae that an automatic extraction process usually has.

\section{References}

1. Ashbaugh, D.R.: Quantitative-Qualitative Friction Ridge Analysis: An Introduction to Basic and Advanced Ridgeology. Practical Aspects of Criminal and Forensic Investigation Series. CRC Press Inc. (1999)

2. Berg, M., Krevelt, M., Overmars, M., Scharzkopf, O.: Computational Geometry (Algorithms and Applications). Springer, Heidelberg (1997)

3. Chikkerur, S., Cartwright, A.N., Govindaraju, V.: K-plet and coupled bfs: A graph based fingerprint representation and matching algorithm. In: International Conference on Biometrics (ICB 2006), pp. 309-315 (2006)

4. Fu, X., Liu, C., Bian, J., Feng, J., Wang, H., Mao, Z.: Extended Clique Models: A New Matching Strategy for Fingerprint Recognition. In: International Conference on Biometrics, ICB 2013 (2013)

5. Gago-Alonso, A., Hernández-Palancar, J., Rodríguez-Reina, E., Muñoz-Briseño, A.: Indexing and retrieving in fingerprint databases under structural distortions (2013), doi:10.1016/j.eswa.2012.12.004

6. Jain, A.K., Feng, J.: Latent Palmprint Matching. IEEE Transactions on Pattern Analysis and Machine Intelligence 31(6), 1032-1047 (2009)

7. Liu, E., Jain, A.K., Tian, J.: A Coarse to Fine Minutiae-Based Latent Palmprint Matching. IEEE Transactions on Pattern Analysis and Machine Intelligence (2013), doi:10.1109/TPAMI.2013.39

8. Neurotechnologija Verifinger 4.2 SDK (2004), http://www.neurotechnologija.com/vfsdk.html

9. Wang, R., Ramos, D., Fiérrez, J.: Latent-to-full palmprint comparison based on radial triangulation under forensic conditions. In: International Joint Conference on Biometrics (IJCB 2011), pp. 1-6. IEEE (2011)

10. Wang, R., Ramos, D., Fiérrez, J.: Improving Radial Triangulation-based Forensic Palmprint Recognition According to Point Pattern Comparison by Relaxation. In: International Conference on Biometrics (ICB 2012), pp. 427-432 (2012)

11. Zhu, E., Hancock, E., Ren, P., Yin, J., Zhang, J.: Associating minutiae between distorted fingerprints using minimal spanning tree. In: Campilho, A., Kamel, M. (eds.) ICIAR 2010, Part II. LNCS, vol. 6112, pp. 235-245. Springer, Heidelberg (2010) 